\title{
In Silico Modeling of Coronavirus Disease 2019 Acute Respiratory Distress Syndrome: Pathophysiologic Insights and Potential Management Implications
}

\author{
Anup Das, $\mathrm{PhD}^{1}$; Sina Saffaran, $\mathrm{PhD}^{1}$; Marc Chikhani, FFICM${ }^{2}$; Timothy E. Scott, $\mathrm{PhD}^{3,4}$; Marianna Laviola, $\mathrm{PhD}^{5}$; \\ Nadir Yehya, $\mathrm{MD}^{6}$; John G. Laffey, MD ${ }^{7,8}$; Jonathan G. Hardman, PhD ${ }^{2,5}$; Declan G. Bates, $\mathrm{PhD}^{1}$
}

Objectives: Patients with coronavirus disease 2019 acute respiratory distress syndrome appear to present with at least two distinct phenotypes: severe hypoxemia with relatively well-preserved lung compliance and lung gas volumes (type 1) and a more conventional acute respiratory distress syndrome phenotype, displaying the typical characteristics of the "baby lung" (type 2). We aimed to test plausible hypotheses regarding the pathophysiologic mechanisms underlying coronavirus disease 2019 acute respiratory distress syndrome and to evaluate the resulting implications for ventilatory management.

Design: We adapted a high-fidelity computational simulator, previously validated in several studies of acute respiratory distress syndrome, to: 1) develop quantitative insights into the key pathophysiologic differences between the coronavirus disease 2019 acute respiratory distress syndrome and the conventional acute respiratory distress syndrome and 2) assess the impact of different positive endexpiratory pressure, $\mathrm{FIO}_{2}$, and tidal volume settings.

${ }^{1}$ School of Engineering, University of Warwick, Coventry, United Kingdom. ${ }^{2}$ Nottingham University Hospitals NHS Trust, Nottingham, United Kingdom. ${ }^{3}$ University Hospitals North Midlands, Stoke-On-Trent, United Kingdom.

${ }^{4}$ Department of Military Anaesthesia and Critical Care, Ministry of Defence, Birmingham, United Kingdom.

${ }^{5}$ School of Medicine, University of Nottingham, Nottingham, United Kingdom. ${ }^{6}$ Division of Pediatric Critical Care Medicine, Department of Anesthesiology and Critical Care Medicine, Children's Hospital of Philadelphia and University of Pennsylvania, Philadelphia, PA.

${ }^{7}$ Galway University Hospitals, Galway, Ireland.

${ }^{8}$ School of Medicine, National University of Ireland, Galway, Ireland.

Copyright (c) 2020 The Authors. Published by Wolters Kluwer Health, Inc. on behalf of the Society of Critical Care Medicine. This is an open-access article distributed under the terms of the Creative Commons Attribution-Non Commercial-No Derivatives License 4.0 (CCBY-NC-ND), where it is permissible to download and share the work provided it is properly cited. The work cannot be changed in any way or used commercially without permission from the journal.

Crit Care Exp/ 2020; 2:e0202

DOI: $10.1097 / C C E .0000000000000202$
Setting: Interdisciplinary Collaboration in Systems Medicine Research Network.

Subjects: The simulator was calibrated to represent coronavirus disease 2019 acute respiratory distress syndrome patients with both normal and elevated body mass indices undergoing invasive mechanical ventilation.

Interventions: None.

Measurements and Main Results: An acute respiratory distress syndrome model implementing disruption of hypoxic pulmonary vasoconstriction and vasodilation leading to hyperperfusion of collapsed lung regions failed to replicate clinical data on type 1 coronavirus disease 2019 acute respiratory distress syndrome patients. Adding mechanisms to reflect disruption of alveolar gas-exchange due to the effects of pneumonitis and heightened vascular resistance due to the emergence of microthrombi produced levels of ventilation perfusion mismatch and hypoxemia consistent with data from type 1 coronavirus disease 2019 acute respiratory distress syndrome patients, while preserving close-to-normal lung compliance and gas volumes. Atypical responses to positive end-expiratory pressure increments between 5 and $15 \mathrm{~cm} \mathrm{H}_{2} \mathrm{O}$ were observed for this type 1 coronavirus disease 2019 acute respiratory distress syndrome model across a range of measures: increasing positive end-expiratory pressure resulted in reduced lung compliance and no improvement in oxygenation, whereas mechanical power, driving pressure, and plateau pressure all increased. $\mathrm{FIO}_{2}$ settings based on acute respiratory distress syndrome network protocols at different positive end-expiratory pressure levels were insufficient to achieve adequate oxygenation. Incrementing tidal volumes from 5 to $10 \mathrm{~mL} / \mathrm{kg}$ produced similar increases in multiple indicators of ventilator-induced lung injury in the type 1 coronavirus disease 2019 acute respiratory distress syndrome model to those seen in a conventional acute respiratory distress syndrome model.

Conclusions: Our model suggests that use of standard positive end-expiratory pressure $/ \mathrm{FIO}_{2}$ tables, higher positive end-expiratory pressure strategies, and higher tidal volumes may all be potentially deleterious in type 1 coronavirus disease 2019 acute respiratory 
distress syndrome patients, and that a highly personalized approach to treatment is advisable.

Key Words: acute respiratory distress syndrome; coronavirus disease 2019; mechanical ventilation; ventilator-induced lung injury

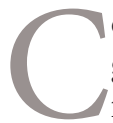

oronavirus disease 2019 (COVID-19) has challenged the global community with a novel disease that is currently not fully understood. As well as the elderly, it has a predilection for the comorbid, particular ethnicities, and the obese. Coronavirus disease 2019 acute respiratory distress syndrome (CARDS) appears to have a dynamic time-related disease spectrum with at least two proposed "phenotypes": a type 1 phenotype (nontypical acute respiratory distress syndrome [ARDS]), characterized by nearnormal lung compliance and pulmonary gas volume, combined with large shunt fraction and severe hypoxemia (1-3), which may evolve into or exist alongside, and a type 2 phenotype characterized by a lower lung compliance ("baby lung" [4]) and a general clinical presentation more typical of severe ARDS. Pulmonary microvascular thrombosis and associated ischemic events also appear to be a particular characteristic of the disease (3).

It is currently unclear whether transitions from the type 1 to type 2 phenotype are due to the natural evolution of the disease, or to the damage to the lung resulting from inappropriate initial ventilatory management leading to a cycle of progressively injurious ventilator-induced lung injury ("VILI vortex") $(5,6)$, or to a combination of the two. Wide variations in mortality rates across different ICUs, together with unexpected patient responses to usual guidelines for ventilatory management, have led some clinicians to assert that standard ventilation protocols employing positive end-expiratory pressure (PEEP)/FIO 2 tables, "open lung" targets, etc. may need significant revision and selective application of their components in CARDS patients (6). A primary concern is that pathophysiologic changes underlying "classical" ARDS ventilation approaches may not apply in some CARDS patients and may thereby lead to ventilator-induced injury, as represented by the conversion of patients from the type 1 to type 2 phenotypes. For example, the use of relatively high PEEP in ARDS treatment is based on the assumption that hypoxemia is due to recruitable nonaerated lung regions; however, in the type 1 CARDS patient, the lung units may be open but nonfunctional due to vascular shunt, and thus PEEP may overdistend (rather than recruit) these lung units, leading to injury without any improvement in gas exchange. The avoidance of VILI in CARDS patients is also of particular importance given the extended time periods for which many of these patients receive mechanical ventilation.

Based on the insights gained from clinical experience, hypotheses have been put forward to explain the particular pathophysiologic characteristics of CARDS patients. Gattinoni et al $(1,2)$ have proposed pulmonary vasoparesis (i.e., globally reduced pulmonary vascular tone) resulting from impaired regulation of lung perfusion and disruption of hypoxic pulmonary vasoconstriction (HPV) as a possible explanation for the severe hypoxemia occurring in the compliant lungs of type 1 patients, whereas results in (7-9) indicate a potentially important role for endothelial damage due to pneumonitis and microthrombi. However, there is currently no direct evidence with which to evaluate quantitatively the effect on gas exchange and ventilation perfusion (V/Q) mismatch of each of these mechanisms, alone or in combination. The clinical evaluation of alternative ventilation strategies for CARDS based on hypothesized models of its pathophysiology is also extremely difficult in the current circumstances.

To shed light on the above issues, we employed a multicompartmental computational model that simulates highly integrated pulmonary and cardiovascular physiologies together with a detailed representation of the effects of invasive mechanical ventilation. This model has been successfully deployed in several previous studies investigating the pathophysiology and ventilatory management of conventional ARDS (10-16). In this study, we use this model to simulate proposed pathophysiologic mechanisms underlying CARDS, by altering key variables to create exemplar patients that reproduce the currently available clinical data. The biomechanics and physiology of obesity are readily amenable to study within this model, and so we include an exemplar patient from this at-risk group. We then use these models to compare the effects of different ventilator settings for CARDS and conventional ARDS patients.

\section{MATERIALS AND METHODS}

\section{Cardiopulmonary Computational Model}

The study employs a multicompartmental computational model that simulates a dynamic in vivo cardiovascular-pulmonary state, comprising conducting airways and a respiratory zone of 100 parallel alveolar compartments, with each compartment having an independent set of variables accounting for stiffness, threshold opening pressures (TOPs), and extrinsic pressures that compress the alveolus as well as airway and perialveolar vascular resistances. This allows for a wide spectrum of ventilation-perfusion mismatch to be replicated. The model includes inherent physiologic reflex mechanisms, for example, HPV. The capability of the simulator to represent the cardiopulmonary disease states of individual patients with ARDS has been validated in a number of previous studies (10-16), and the mathematic and physiologic principles on which it is based are detailed in the Supplementary Material, http://links.lww.com/CCX/A295.

\section{Measurements of Key Physiologic Variables and VILI Indices From the Model}

Mechanical ventilation in the model is set to pressure-controlled mode with respiratory rate at 20 beats/min, inspiratoryto-expiratory ratio at $1: 2$, and $\mathrm{FIO}_{2}$ set to $100 \%$. To observe the cardiopulmonary effects of interest, the following values were computed and recorded: $\mathrm{PaO}_{2}, \mathrm{PaCO}_{2}$, arterial $\mathrm{pH}$, arterial oxygen saturation $\left(\mathrm{SaO}_{2}\right)$, mixed venous oxygen saturation, volume of individual alveolar compartments at end-inspiration $\left(\mathrm{V}_{\mathrm{alv} \_ \text {insp }}\right)$, volume of individual alveolar compartments at end-expiration $\left(\mathrm{V}_{\text {alv_exp }}\right)$, pressure of individual alveolar compartments at endinspiration $\left(\mathrm{P}_{\text {alv_insp }}\right)$, pressure of individual alveolar compartments at end-expiration $\left(\mathrm{P}_{\text {alv_exp }}\right)$, cardiac output $(\mathrm{CO})$, mean arterial pressure, mean pulmonary artery pressure, and arterial oxygen delivery $\left(\mathrm{DO}_{2}\right.$; computed using the values of $\mathrm{CO}, \mathrm{SaO}_{2}$, and $\mathrm{PaO}_{2}$ 
and hemoglobin level). Alveolar recruitment was calculated as the fraction of alveoli receiving zero ventilation and subsequently achieving ventilation. VILI indices calculated include respiratory system compliance, intratidal recruitment, mechanical power (16), driving pressure, mean alveolar pressure, and dynamic strain (calculated as $\Delta \mathrm{V} / \mathrm{V}_{\text {frc }}$, where $\mathrm{V}_{\text {frc }}$ is $\mathrm{V}_{\text {alveexp }}$ at $\mathrm{PEEP}=0 \mathrm{~cm} \mathrm{H}_{2} \mathrm{O}$ and $\Delta \mathrm{V}=\mathrm{V}_{\text {alv_insp }}-\mathrm{V}_{\text {alv_exp }}$ ).

Intratidal recruitment was calculated as the difference between the ratio of total ventilated lung at the end of inhalation to that at the end of exhalation. Shunt fraction $\left(\mathrm{Q}_{\mathrm{S}} / \mathrm{Q}_{\mathrm{T}}\right)$ was calculated using the shunt equation, based on the arterial, pulmonary end-capillary, and mixed venous oxygen content. Physiologic dead space volume and tidal volume $(\mathrm{VD} / \mathrm{VT})$ were calculated from $\mathrm{PaCO}_{2}$, mixed expired $\mathrm{CO}_{2}$ tension, and exhaled $\mathrm{VT}$ (17). $\mathrm{PEEP}_{\text {тот }}$ is measured as the total pressure within the lung at the end of expiration and accounts for both the extrinsic applied expiratory pressure and intrinsically generated end-expiratory pressures. Plateau pressure (Pplat) is measured as the average end-inspiratory pressure in the lung. Lung compliance is calculated as $\Delta \mathrm{V} / \Delta \mathrm{P}$, where $\Delta \mathrm{P}$ is the Pplat minus $\mathrm{PEEP}_{\mathrm{TOT}}$ All simulations were run for 30 minutes, and during simulations, all variables were updated and recorded every $10 \mathrm{~ms}$.

\section{RESULTS}

\section{Pathophysiologic Mechanisms That Produce a Type 1 CARDS Phenotype}

We configured the simulator's baseline settings to represent two "exemplar" patients-a typical (ideal body weight) 70-kg patient and an obese patient weighing $110 \mathrm{~kg}$ (body mass indices [BMIs] 24 and $37.7 \mathrm{~kg} / \mathrm{m}^{2}$, respectively). Patients were assumed to be sedated and to be receiving positive pressure mechanical ventilation without any spontaneous ventilatory effort.

The exemplar patient models were then configured according to clinical data on different CARDS phenotypes available in the literature. Based on the data (1-6) suggesting that type 1 CARDS patients have relatively well-preserved lung gas volume and compliance, the type 1 models were set to have $8 \%$ (11\% in patients with elevated BMI) of their alveoli collapsed, that is, nonaerated, with low recruitability (i.e., extrinsic pressures acting on collapsed alveolar compartments were set to greater than $40 \mathrm{~cm} \mathrm{H}_{2} \mathrm{O}$ with average TOPs of $50 \mathrm{~cm} \mathrm{H}_{2} \mathrm{O}$ ) (1-4). To simulate the hyperperfusion of gasless tissue reported in the study by Gattinoni et al (1), we implemented vasodilation in the collapsed units by decreasing their vascular resistance by $80 \%$. HPV is normally incorporated in our simulator via a mathematic function, based on the stimulus-response curve suggested in (18); to simulate the hypothesized disruption of HPV in CARDS, we disabled this function in our model. Simulating the effects of these mechanisms alone did not produce levels of $\mathrm{Q}_{\mathrm{S}} / \mathrm{Q}_{\mathrm{T}}$, dead space, and hypoxemia matching those reported in clinical data describing type 1 CARDS (Table 1). We, therefore, also incorporated disruption of alveolar gas-exchange due to the effects of pneumonitis into the model by blocking alveolar-capillary gas equilibration in $30 \%$ of the alveolar compartments. As thrombotic complications have been reported to be a characteristic feature of CARDS, we modeled the presence of microthrombi by increasing vascular resistance by a factor of 100 in 10 of the 30 compartments with disrupted gas exchange. Implementing the above additional pathophysiologic mechanisms in our model produced levels of $\mathrm{Q}_{\mathrm{S}} / \mathrm{Q}_{\mathrm{T}}(47.8 \%)$, dead space $(192.3 \mathrm{~mL})$, and hypoxemia $\left(\mathrm{SaO}_{2} 87.6 \%\right.$ and $\mathrm{FiO}_{2}$ of $\left.100 \%\right)$ that match those reported in clinical data describing type 1 CARDS (1-6), while still preserving near-normal levels of lung compliance $\left(43.5 \mathrm{~mL} / \mathrm{cm} \mathrm{H}_{2} \mathrm{O}\right.$ ) (Table 1 ).

Although a reasonable assumption, there is currently no clear evidence that pulmonary vascular microthrombi primarily arise in alveoli that have been damaged by pneumonitis, and therefore, we also modeled the effect of microthrombi being heterogeneously distributed throughout the lung, by increasing vascular resistance in five of the disrupted and five of the normally functioning compartments. This further worsened V/Q matching $\left(\mathrm{Q}_{\mathrm{S}} / \mathrm{Q}_{\mathrm{T}}\right.$ increased to $51.8 \%$ and dead space to $205.9 \mathrm{~mL}$ ) leading to severe hypoxemia $\left(\mathrm{SaO}_{2} 83.8 \%\right.$ and $\mathrm{FiO}_{2}$ of $\left.100 \%\right)$ (Table 1).

For the purposes of comparison, both normal and elevated BMI patients were also configured to represent a conventional ARDS phenotype $(3,4)$. Specifically, the compliance and TOPs of 50 alveolar compartments were configured to create nonaerated (at baseline) but recruitable lung regions (extrinsic pressures that induce collapse, set with a mean value of $20 \mathrm{~cm} \mathrm{H}_{2} \mathrm{O}$ and a mean TOP of $20 \mathrm{~cm} \mathrm{H}_{2} \mathrm{O}$ ), resulting in a $\mathrm{Q}_{\mathrm{S}} / \mathrm{Q}_{\mathrm{T}}$ of $39 \%$, VD/VT of 0.3 , and compliance of $35.1 \mathrm{~mL} / \mathrm{cm} \mathrm{H}_{2} \mathrm{O}$ in the normal BMI model.

\section{Effects of High Versus Low PEEP}

Figure 1 shows the results of reducing PEEP to $5 \mathrm{~cm} \mathrm{H}_{2} \mathrm{O}$ and increasing PEEP to $15 \mathrm{~cm} \mathrm{H}_{2} \mathrm{O}$ (from a baseline setting of $10 \mathrm{~cm}$ $\mathrm{H}_{2} \mathrm{O}$ ), while keeping Vт fixed at $7 \mathrm{~mL} / \mathrm{kg}$, for both the type 1 CARDS and conventional ARDS models (normal BMIs). As shown, strikingly different responses were observed for the two models across a range of measures. For the conventional ARDS model, increasing PEEP produced improvements in $\mathrm{Do}_{2}$ and arterial oxygenation $\left(\mathrm{PaO}_{2}\right.$ and $\left.\mathrm{SaO}_{2}\right)$ resulting from increased alveolar recruitment, increased lung compliance, and reduced shunt, with a reduction in driving pressure and only a modest increase in mechanical power. In contrast, for the type 1 CARDS model, increasing PEEP reduced $\mathrm{Do}_{2}$, produced no improvement in oxygenation, and reduced lung compliance, whereas mechanical power, driving pressure, and Pplat were all increased. In the obese type 1 CARDS patient, increasing PEEP did slightly increase $\mathrm{Do}_{2}$ and arterial oxygenation, but to a lesser extent than in the conventional ARDS model; see Figure S1 in the Supplementary Material, http://links.lww.com/CCX/A295. Corresponding results in terms of relative changes, rather than absolute values, for both figures are shown in Figures S2 and S3 of the Supplementary Material, http:// links.lww.com/CCX/A295.

\section{Effects of Applying Acute Respiratory Distress Syndrome Network PEEP/FıO ${ }_{2}$ Tables}

To investigate the utility of applying standard combinations of PEEP and $\mathrm{FIO}_{2}$ based on the tables derived from the acute respiratory distress syndrome network (ARDSnet) trial (19), we evaluated the effect of varying $\mathrm{FIO}_{2}$ between 0.4 and 1 at the PEEP levels of 5 and $10 \mathrm{~cm} \mathrm{H}_{2} \mathrm{O}$. In the type 1 CARDS model, at a PEEP of 
TABLE 1. Pathophysiological Mechanisms Generating a Type 1 Coronavirus Disease 2019 Acute Respiratory Distress Syndrome Phenotype

\begin{tabular}{|c|c|c|c|c|c|c|c|}
\hline & \multicolumn{4}{|c|}{ Normal BMI } & \multicolumn{3}{|c|}{ Elevated BMI } \\
\hline & & $\begin{array}{l}\text { acollocated } \\
\text { Pneumonitis and } \\
\text { Thrombosis }\end{array}$ & $\begin{array}{l}\text { aDispersed Pneur } \\
\text { Thrombosis (5\% } \\
\text { in Pneumoniti } \\
\text { Thrombosis in Non } \\
\text { Compartm }\end{array}$ & $\begin{array}{l}\text { monitis and } \\
\text { Thrombosis } \\
\text { is and } 5 \% \\
\text { npneumonitis } \\
\text { lents) }\end{array}$ & $\begin{array}{l}\text { aCollocated } \\
\text { Pneumonitis and } \\
\text { Thrombosis }\end{array}$ & $\begin{array}{r}\text { aDispersed Pneu } \\
\text { Thrombosis (5\% } \\
\text { in Pneumoniti } \\
\text { Thrombosis in No } \\
\text { Compartm }\end{array}$ & $\begin{array}{l}\text { monitis and } \\
\text { Thrombosis } \\
\text { s and } 5 \% \\
\text { ipneumonitis } \\
\text { ents) }\end{array}$ \\
\hline & $\begin{array}{c}\text { Type } 1 \\
\text { Phenotype } \\
\text { (Vasodilation } \\
\text { HPV Off) }\end{array}$ & $\begin{array}{c}\text { Type } 1 \\
\text { Phenotype } \\
\text { (Pneumonitis 30\%, } \\
\text { Thrombosis } \\
10 \% \text {, and } \\
\text { Vasodilation } \\
\text { HPV Off) }\end{array}$ & $\begin{array}{c}\text { Type } 1 \\
\text { Phenotype } \\
\text { (Pneumonitis } \\
\text { 30\%, Thrombosis } \\
\text { 10\%, and } \\
\text { Vasodilation } \\
\text { HPV Off) }\end{array}$ & $\begin{array}{c}\text { Conventional } \\
\text { ARDS }\end{array}$ & $\begin{array}{c}\text { Type } 1 \\
\text { Phenotype } \\
\text { (Pneumonitis } \\
\text { 30\%, Thrombosis } \\
\text { 10\%, and } \\
\text { Vasodilation } \\
\text { HPV Off) }\end{array}$ & $\begin{array}{c}\text { Type } 1 \\
\text { Phenotype } \\
\text { (Pneumonitis } \\
30 \% \text {, Thrombosis } \\
\text { 10\%, and } \\
\text { Vasodilation } \\
\text { HPV Off) }\end{array}$ & $\begin{array}{l}\text { Conventional } \\
\text { ARDS }\end{array}$ \\
\hline $\begin{array}{l}\text { Positive end- } \\
\text { expiratory } \\
\text { pressure, } \mathrm{cm} \mathrm{H}_{2} \mathrm{O}\end{array}$ & 5 & 5 & 5 & 5 & 5 & 5 & 5 \\
\hline $\begin{array}{l}\text { Ventilation rate, } \\
\text { beats/min }\end{array}$ & 20 & 20 & 20 & 20 & 20 & 20 & 20 \\
\hline Duty cycle & 0.33 & 0.33 & 0.33 & 0.33 & 0.33 & 0.33 & 0.33 \\
\hline $\mathrm{FlO}_{2}$ & 1 & 1 & 1 & 1 & 1 & 1 & 1 \\
\hline $\mathrm{V}_{\mathrm{T}}, \mathrm{mL}$ & 470 & 470 & 470 & 470 & 490 & 490 & 490 \\
\hline $\mathrm{V}_{\mathrm{T}}, \mathrm{mL} / \mathrm{kg}$ & 6.7 & 6.7 & 6.7 & 6.7 & 7.0 & 7.0 & 7.0 \\
\hline $\begin{array}{l}\text { Peak pressure, cm } \\
\mathrm{H}_{2} \mathrm{O}\end{array}$ & 25 & 25 & 25 & 30 & 25 & 25 & 30 \\
\hline Shunt fraction, \% & 28.0 & 47.78 & 51.79 & 39.01 & 52.05 & 59.75 & 42.92 \\
\hline $\mathrm{VD}_{\mathrm{D}} \mathrm{mL}$ & 118.3 & 192.3 & 205.2 & 142.9 & 220.9 & 234.4 & 172.8 \\
\hline $\mathrm{VD}_{\mathrm{D}} \mathrm{V}_{\mathrm{T}}$ & 0.25 & 0.4 & 0.43 & 0.3 & 0.45 & 0.48 & 0.35 \\
\hline $\begin{array}{l}\text { Respiratory system } \\
\text { compliance, } \\
\mathrm{mL} / \mathrm{cm} \mathrm{H}_{2} \mathrm{O}\end{array}$ & 43.6 & 43.28 & 43.28 & 35.05 & 40.65 & 39.98 & 32.03 \\
\hline $\begin{array}{l}\text { Arterial oxygen } \\
\text { saturation, } \%\end{array}$ & 96.6 & 87.6 & 83.79 & 90.3 & 84.18 & 75.14 & 87.69 \\
\hline $\mathrm{PaO}_{2}, \mathrm{kPa}$ & 10.9 & 7.5 & 6.9 & 8.42 & 7.41 & 6.27 & 8.36 \\
\hline $\begin{array}{l}\text { Ratio of } \mathrm{PaO}_{2} \text { to } \\
\mathrm{FlO}_{2}, \mathrm{~mm} \mathrm{Hg}\end{array}$ & 117.0 & 56.5 & 51.75 & 63.15 & 55.58 & 47.02 & 62.7 \\
\hline $\mathrm{PacO}_{2}, \mathrm{kPa}$ & 4.4 & 6.1 & 6.5 & 6.1 & 7.9 & 8.7 & 8 \\
\hline $\begin{array}{l}\text { Cardiac output, } \\
\text { L/min }\end{array}$ & 6.3 & 6.21 & 6.21 & 6.04 & 7.81 & 7.81 & 7.36 \\
\hline $\begin{array}{l}\text { Mean pulmonary } \\
\text { artery pressure, } \\
\text { mm Hg }\end{array}$ & 19 & 20.18 & 20.18 & 25.25 & 23.58 & 23.57 & 30.18 \\
\hline $\begin{array}{l}\text { Mean arterial } \\
\quad \text { pressure, } \mathrm{mm} \mathrm{Hg}\end{array}$ & 108.5 & 125.32 & 125.32 & 123.87 & 132.91 & 132.92 & 128.17 \\
\hline $\begin{array}{l}\text { Mechanical power, } \\
\mathrm{J} / \mathrm{min}\end{array}$ & 17.18 & 17.14 & 17.14 & 19.96 & 17.51 & 17.25 & 20.14 \\
\hline Dynamic strain & 0.17 & 0.17 & 0.17 & 0.17 & 0.29 & 0.28 & 0.29 \\
\hline $\begin{array}{l}\text { Driving pressure, } \\
\mathrm{cm} \mathrm{H}_{2} \mathrm{O}\end{array}$ & 12.9 & 12.9 & 12.9 & 16.6 & 13.8 & 13.8 & 17.9 \\
\hline
\end{tabular}

$\mathrm{ARDS}=$ acute respiratory distress syndrome, $\mathrm{BMI}=$ body mass index, $\mathrm{HPV}=$ hypoxic pulmonary vasoconstriction, $\mathrm{V}_{\mathrm{D}}=$ dead space volume, $\mathrm{V}_{T}=$ tidal volume. aThe description of the pathophysiology. 


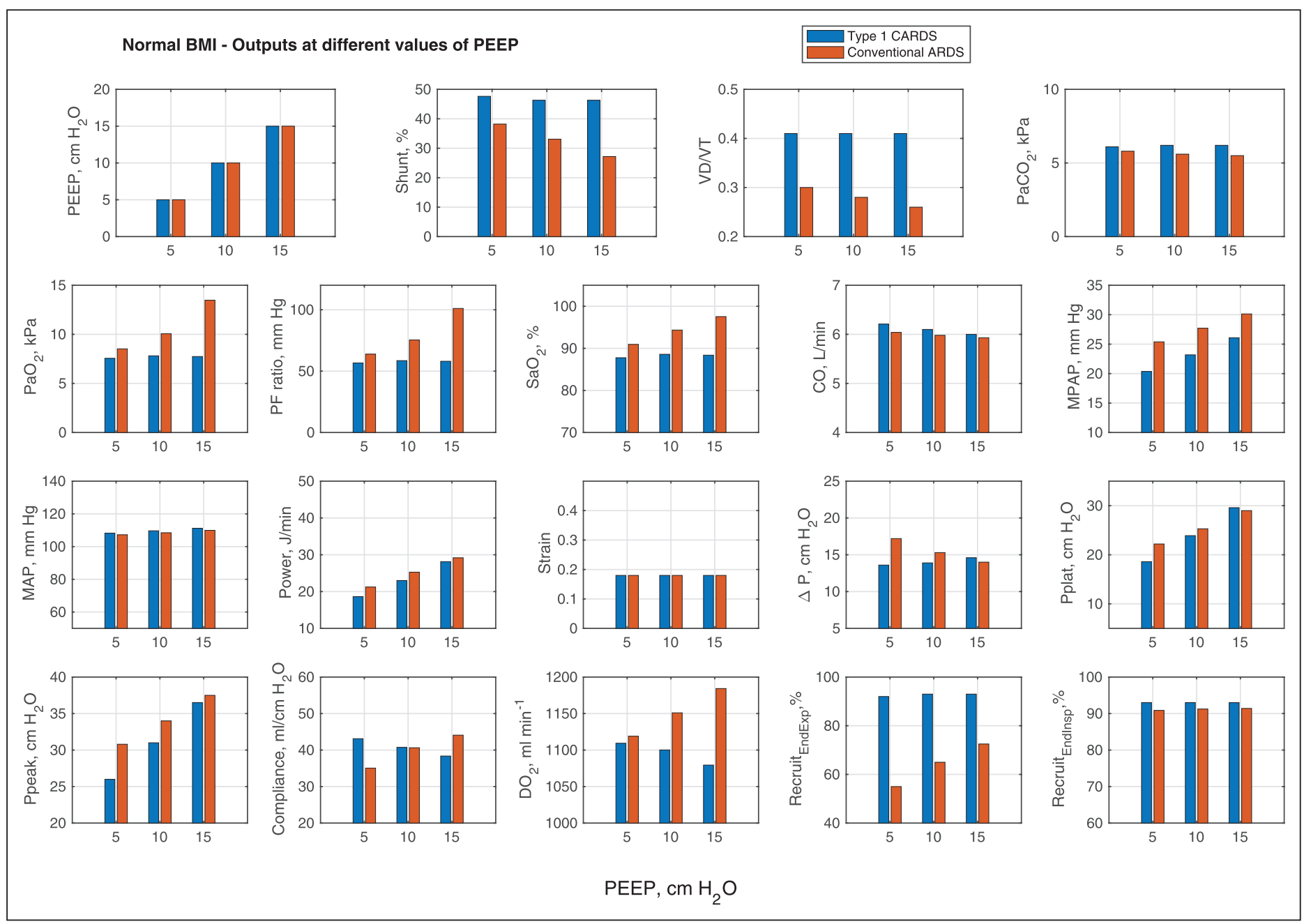

Figure 1. Results of reducing positive end-expiratory pressure (PEEP) to $5 \mathrm{~cm} \mathrm{H} \mathrm{H}_{2} \mathrm{O}$ and increasing PEEP to $15 \mathrm{~cm} \mathrm{H}_{2} \mathrm{O}$ (from a baseline setting of $10 \mathrm{~cm} \mathrm{H}_{2} \mathrm{O}$ ), while keeping tidal volume $(\mathrm{VT})$ fixed at $7 \mathrm{~mL} / \mathrm{kg}$, for both the type 1 coronavirus disease 2019 acute respiratory distress syndrome (CARDS) and conventional

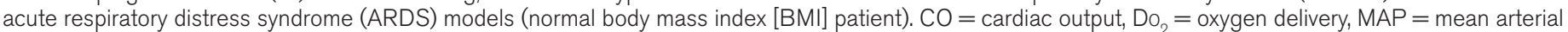
pressure, $\mathrm{MPAP}=$ mean pulmonary artery pressure, $\mathrm{PF}=$ ratio of $\mathrm{PaO}_{2}$ to $\mathrm{FIO}_{2}$, power $=$ mechanical power, Ppeak $=$ peak pressure, $\mathrm{Pplat}=$ plateau pressure, $\mathrm{SaO}_{2}=$ arterial oxygen saturation, strain $=$ dynamic strain, $\mathrm{VD}=$ dead space volume, $\Delta \mathrm{P}=$ driving pressure.

$5 \mathrm{~cm} \mathrm{H}_{2} \mathrm{O}$, application of an $\mathrm{FIO}_{2}$ of 0.4 (corresponding to the top of the range suggested in [19]) is insufficient to produce a level of $\mathrm{PaO}_{2}$ exceeding $7.3 \mathrm{kPa}$ (Fig. 2). At a PEEP of $10 \mathrm{~cm} \mathrm{H}_{2} \mathrm{O}$, application of $\mathrm{FIO}_{2}$ of 0.7 as suggested in (19) is also insufficient to ensure adequate oxygenation in the type 1 CARDS model. In contrast, application of these settings to the conventional ARDS model produces $\mathrm{PaO}_{2}$ levels above the $7.3 \mathrm{kPa}$ threshold for both PEEP values.

\section{Effects of High Versus Low $V_{T}$}

Figure 3 shows the results of reducing VT to $5 \mathrm{~mL} / \mathrm{kg}$ and increasing VT to $10 \mathrm{~mL} / \mathrm{kg}$ (from a baseline setting of $7 \mathrm{~mL} / \mathrm{kg}$ ), while keeping PEEP fixed at $10 \mathrm{~cm} \mathrm{H}_{2} \mathrm{O}$, for both the type 1 CARDS and conventional ARDS models (normal BMIs). In this case, increased Vт does produce some improvements in gas exchange in the type 1 CARDS model, with increased $\mathrm{SaO}_{2}$ and $\mathrm{Do}_{2}$ and reduced $\mathrm{PaCO}_{2}$, although the improvements are smaller than those seen in the conventional ARDS model. Notably, however, the associated worsening in VILI indicators (mechanical power, driving pressure, dynamic strain, and Pplat) mirrors or exceeds that produced in the conventional ARDS model. Increasing VT in the obese models also produced improved gas exchange, but again at the cost of clinically significant increases in multiple VILI indices; see Figure S4 in the Supplementary Material, http://links. lww.com/CCX/A295. Corresponding results in terms of relative changes, rather than absolute values, for both figures are shown in Figures S5 and S6 of the Supplementary Material, http://links. lww.com/CCX/A295.

\section{DISCUSSION}

The modifications required to our conventional ARDS model to allow it to reproduce the particular features of type 1 patients are consistent with recent data on lung perfusion abnormalities in CARDS patients from imaging and postmortem studies. In the study by Lang et al (20), dual-energy CT imaging of three CARDS patients revealed preferentially increased perfusion of the lungs proximal to areas of lung opacity, decreased areas of peripheral perfusion corresponding to peripheral lung opacities, and a halo of increased perfusion surrounding peripheral areas 


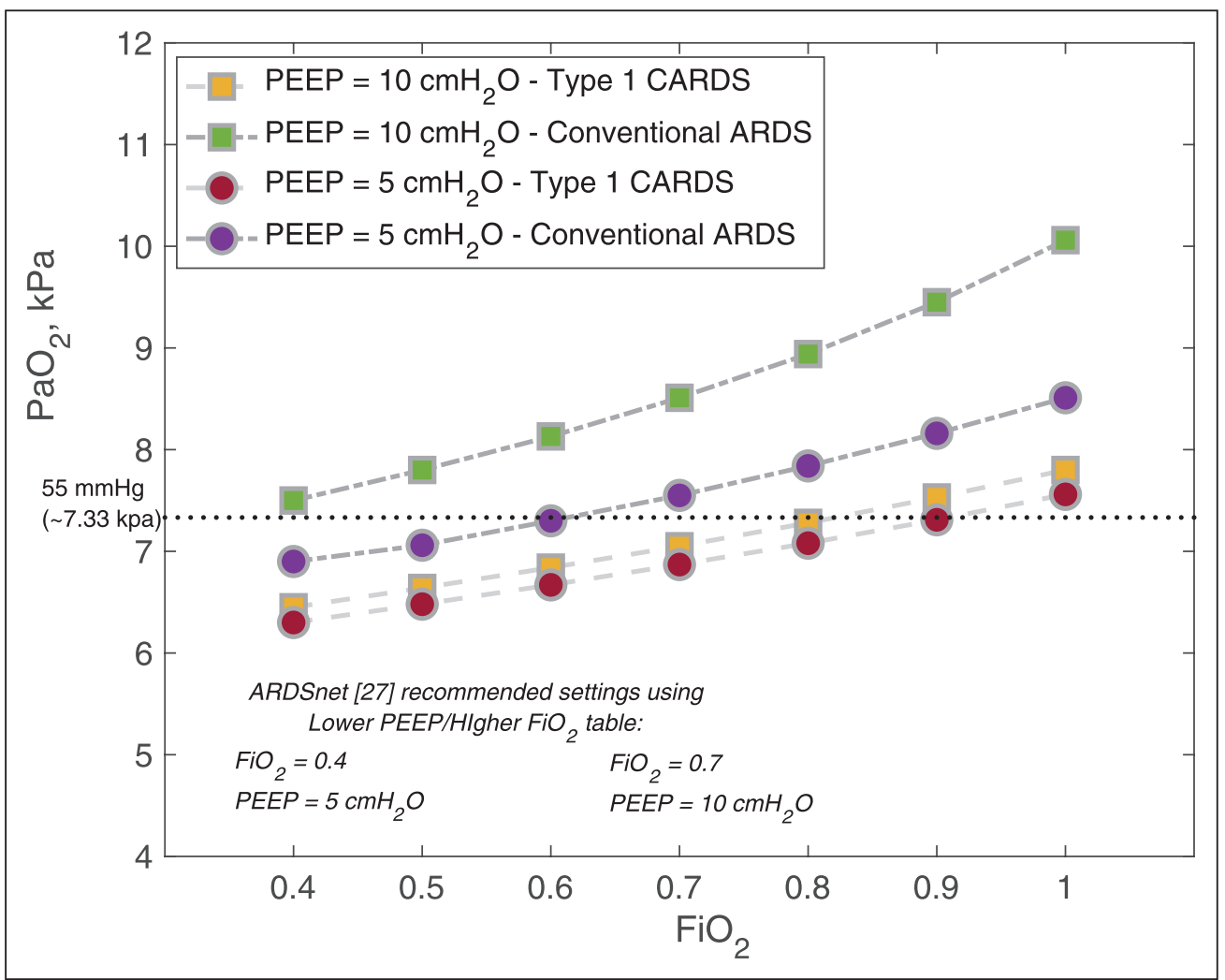

Figure 2. Results of varying $\mathrm{FIO}_{2}$ at positive end-expiratory pressure (PEEP) levels of 5 and $10 \mathrm{~cm} \mathrm{H}_{2} \mathrm{O}$ in both the type 1 coronavirus disease 2019 acute respiratory distress syndrome (CARDS) and conventional acute respiratory distress syndrome (ARDS) models. ARDSnet = acute respiratory distress syndrome network. reveal a wide variation in the level of recruitability, consistent with our results. Data from these studies suggest that there is potential for PEEPinduced VILI in nonrecruitable CARDS lungs (higher driving pressures due to increased PEEP in [24] and reduced compliance in [25]), and this is confirmed by the marked elevation in multiple VILI indicators in our type 1 CARDS model as PEEP is increased from 5 to $15 \mathrm{~cm}$ $\mathrm{H}_{2} \mathrm{O}$. Based on these results, and as recently argued in (26), the use of high PEEP in type 1 CARDS patients does not appear to yield a favorable risk/benefit profile; it is, therefore, essential to evaluate the recruitability of each patient carefully before employing a standard ARDSnet low VT/high PEEP strategy. As demonstrated in Figure 2, application of standard ARDSnet type PEEP/FIO tables in type 1 CARDS patients is also unlikely to achieve the levels of oxygenation typically produced in conventional ARDS patients.

It has been asserted that CARDS patients may be able to accept larger of consolidation. A remarkable level of hyperperfusion of gasless tissue was also noted in (1). A postmortem study reported vasodilation of pulmonary vessels in two CARDS patients (21). Finally, two recent studies indicated that CARDS patients are prone to significant vascular dysfunction (also confirmed by the authors' clinical experience): in the study by Helms et al (7), 64 clinically relevant thrombotic complications were diagnosed in 150 CARDS patients (including pulmonary embolisms) with greater than $95 \%$ of patients having elevated D-dimer and fibrinogen, whereas a postmortem examination of 21 CARDS patients in the study by Menter et al (8) reported massive capillary congestion often accompanied by microthrombi and pulmonary emboli, despite prophylactic anticoagulation.

In our model, we found that implementing pulmonary vasoparesis on its own was not sufficient to produce the levels of shunt and hypoxia, etc. seen in type 1 patients. However, combining this mechanism with pneumonitis-induced disruption of the alveolarcapillary barrier (as proposed in [9]) and the presence of microthrombi (as reported in $[7,8]$ ) allows the model to produce the observed levels of V/Q mismatch and hypoxia, while preserving normal lung compliance and gas volumes. Interestingly, analysis of data from the LUNG SAFE study (22) indicates that a subset of patients with type 1 characteristics (e.g., poor PEEP responsiveness) also exists in "classical" ARDS; however, their frequency is lower than that seen in CARDS.

Several studies have examined lung recruitability in small cohorts of CARDS patients using PEEP titration (23-25). Results
VTs than those now normally applied in lung protective ventilation for ARDS without increasing the risk of VILI (5). Our results indicate that use of higher $V \mathrm{~T}$ can produce improvements in oxygenation, but the observed worsening of VILI indicators raises concerns that higher VT could also damage the type 1 CARDS lung. This may be of particular importance given the relatively longer periods for which CARDS patients appear to require mechanical ventilation (27). Given the stronger association between improved driving pressure and outcome (28), relative to improved gas exchange (19), the risks of increasing Vт to improve hypoxemia need to be carefully balanced against the increased mortality risk due to higher driving pressure.

The application of prone positioning in patients with CARDS (whether mechanically or noninvasively ventilated) has, in the clinical experience of the authors, a significant beneficial effect on the ratio of $\mathrm{PaO}_{2}$ to $\mathrm{FiO}_{2}(\mathrm{P} / \mathrm{F})$ and, albeit anecdotally, on patient outcomes. Globally, intensive care teams are unlikely to have ever simultaneously looked after as many proned patients as they have during the COVID-19 outbreak, and arguably, the management of such patients combined with the logistical burden this entailed will be the hallmark of the period. Already of proven benefit in patients with hypoxic respiratory failure (29), prone positioning may have an even more pronounced effect in patients requiring mechanical ventilation due to CARDS. The maneuver has been so successful, at least in the short term, that prone positioning has been extrapolated to spontaneously breathing patients (for the first time in the authors' experience), resulting in, anecdotally, equally impressive improvements in oxygenation. Prolonged prone positioning has 


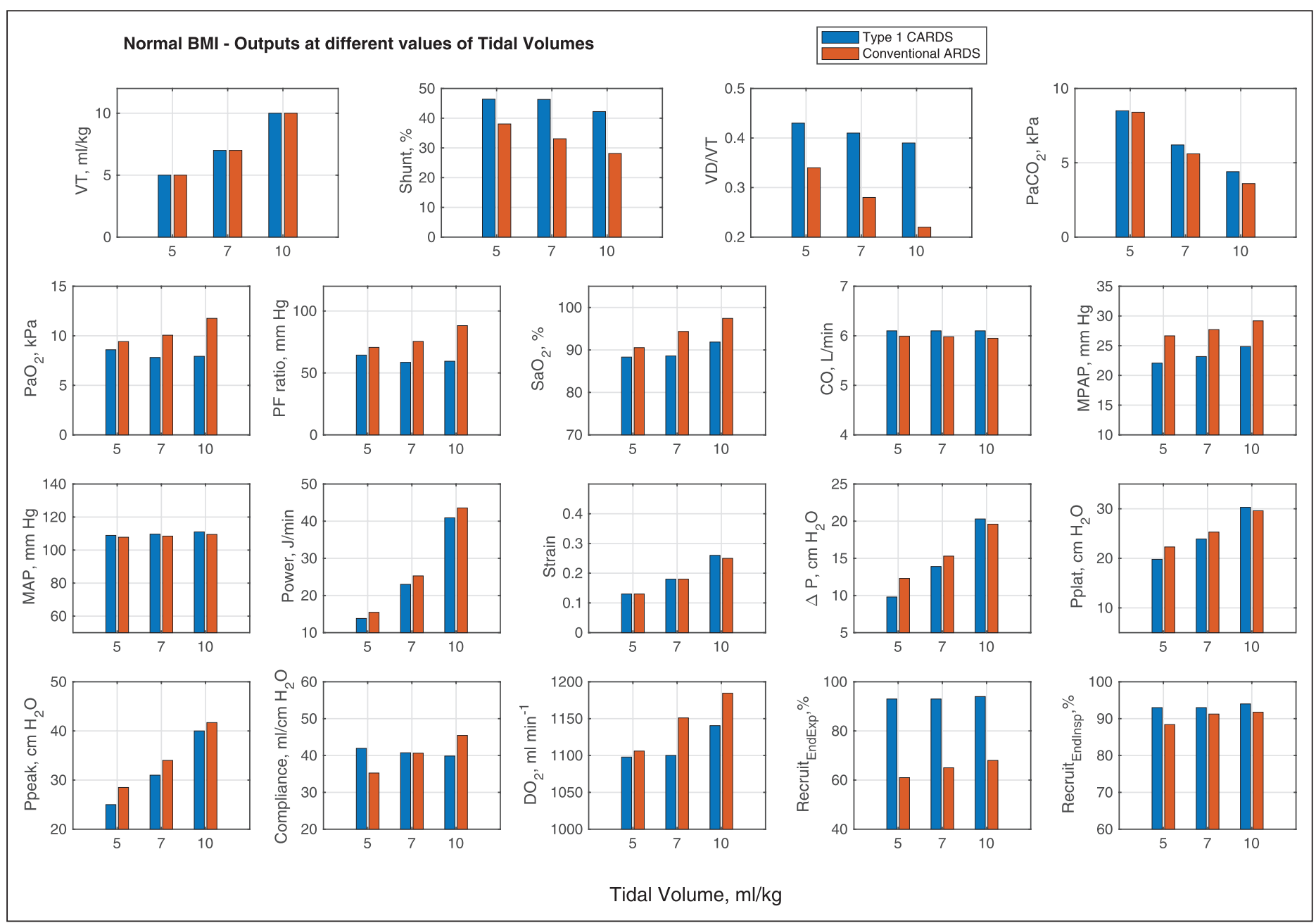

Figure 3. Results of reducing tidal volume $\left(\mathrm{VT}_{\mathrm{T}}\right)$ to $5 \mathrm{~mL} / \mathrm{kg}$ and increasing $\mathrm{V}_{\mathrm{T}}$ to $10 \mathrm{~mL} / \mathrm{kg}$ (from a baseline setting of $7 \mathrm{~mL} / \mathrm{kg}$ ), while keeping positive endexpiratory pressure (PEEP) fixed at $10 \mathrm{~cm} \mathrm{H}_{2} \mathrm{O}$, for both the type 1 and conventional acute respiratory distress syndrome (ARDS) models (normal body mass index [BMI] patient). CARDS = coronavirus disease 2019 acute respiratory distress syndrome, $\mathrm{CO}=$ cardiac output, $\mathrm{Do}_{2}=\mathrm{oxygen}_{\mathrm{g}}$ delivery, $\mathrm{MAP}=$ mean arterial pressure, MPAP = mean pulmonary artery pressure, $\mathrm{PF}=$ ratio of $\mathrm{PaO}_{2}$ to $\mathrm{FIO}_{2}$, power $=$ mechanical power, Ppeak $=$ peak pressure, Pplat $=$ plateau pressure, $\mathrm{SaO}_{2}=$ arterial oxygen saturation, strain $=$ dynamic strain, $\mathrm{VD}_{\mathrm{D}}=$ dead space volume, $\Delta \mathrm{P}=$ driving pressure.

recently been demonstrated to result in improvements in $\mathrm{P} / \mathrm{F}$ ratios to greater than those recorded preintubation in a small study of CARDS patients, with no change in the complication rate (30).

From a physiologic standpoint, quite why proning might be so effective in CARDS is not currently understood, particularly in the type 1 phenotype, where its use remains controversial (1-5). The argument that proning improves V/Q mismatch through increasing blood flow to functioning ventral alveoli seems to fall short of a full explanation for the significant improvements seen in oxygenation in CARDS. While dual-energy CT scanning of such patients is technically feasible, it is logistically difficult. Use of a physiologic simulator such as that developed here may allow us to understand the mechanistic basis for the improvements achieved by proning, and potentially guide the timing, duration, and optimization of ventilation for prone positioned CARDS patients.

\section{CONCLUSIONS}

A model implementing: 1) vasodilation leading to hyperperfusion of collapsed lung regions, 2) disruption of HPV, 3) disruption of alveolar gas-exchange due to the effects of pneumonitis, and 4) heightened vascular resistance due to the presence of microthrombi replicates levels of ventilation-perfusion mismatch and hypoxemia exhibited by type 1 CARDS patients, while preserving close-to-normal lung compliance and gas volumes. Our results suggest that ventilatory management of such patients (whether with normal or elevated BMI) should not follow standard protocols, but rather focus on keeping PEEP, and where possible Vт, low, in order to avoid the risk of entering the VILI vortex.

Supplemental digital content is available for this article. Direct URL citations appear in the printed text and are provided in the HTML and PDF versions of this article on the journal's website (http://journals.lww.com/ccejournal).

Supported, in part, by the U.K. Engineering and Physical Sciences Research Council (grants EP/P023444/1 and EP/V014455/1).

The authors have disclosed that they do not have any potential conflicts of interest.

For information regarding this article, E-mail: j.hardman@nottingham.ac.uk; D.Bates@warwick.ac.uk 


\section{REFERENCES}

1. Gattinoni L, Coppola S, Cressoni M, et al: Covid-19 does not lead to a "typical" acute respiratory distress syndrome. Am J Respir Crit Care Med 2020; 201:1299-1300

2. Gattinoni L, Chiumello D, Caironi P, et al: COVID-19 pneumonia: Different respiratory treatment for different phenotypes? Int Care Med 2020; 46:1099-1102

3. Gattinoni L, Chiumello D, Rossi S: COVID-19 pneumonia: ARDS or not? Crit Care 2020; 24:154

4. Gattinoni L, Meissner K, Marini JJ: The baby lung and the COVID-19 era. Int Care Med 2020; 46:1438-1440

5. Marini JJ, Gattinoni L: Management of COVID-19 respiratory distress. JAMA 2020; 323:2329-2330

6. Marini JJ: Dealing with the CARDS of COVID-19. Crit Care Med 2020; 48:1239-1241

7. Helms J, Tacquard C, Severac F, et al; CRICS TRIGGERSEP Group (Clinical Research in Intensive Care and Sepsis Trial Group for Global Evaluation and Research in Sepsis): High risk of thrombosis in patients with severe SARS-CoV-2 infection: A multicenter prospective cohort study. Intensive Care Med 2020; 46:1089-1098

8. Menter T, Haslbauer JD, Nienhold R, et al: Postmortem examination of COVID-19 patients reveals diffuse alveolar damage with severe capillary congestion and variegated findings of lungs and other organs suggesting vascular dysfunction. Histopathology 2020 May 4. [online ahead of print]

9. Jain A, Doyle DJ: Stages or phenotypes? A critical look at COVID-19 pathophysiology. Int Care Med 2020; 46:1494-1495

10. McCahon RA, Columb MO, Mahajan RP, et al: Validation and application of a high-fidelity, computational model of acute respiratory distress syndrome to the examination of the indices of oxygenation at constant lung-state. Br J Anaesth 2008; 101:358-365

11. Das A, Cole O, Chikhani M, et al: Evaluation of lung recruitment maneuvers in acute respiratory distress syndrome using computer simulation. Crit Care 2015; 19:8

12. Chikhani M, Das A, Haque M, et al: High PEEP in acute respiratory distress syndrome: Quantitative evaluation between improved arterial oxygenation and decreased oxygen delivery. Br J Anaesth 2016; 117:650-658

13. Das A, Haque M, Chikhani M, et al: Hemodynamic effects of lung recruitment maneuvers in acute respiratory distress syndrome. BMC Pulm Med 2017; 17:34

14. Das A, Camporota L, Hardman JG, et al: What links ventilator driving pressure with survival in the acute respiratory distress syndrome? A computational study. Respir Res 2019; 20:29

15. Saffaran S, Das A, Hardman JG, et al: High-fidelity computational simulation to refine strategies for lung-protective ventilation in paediatric acute respiratory distress syndrome. Int Care Med 2019; 45:1055-1057
16. Saffaran S, Das A, Laffey JG, et al: Utility of driving pressure and mechanical power to guide protective ventilator settings in two cohorts of adult and pediatric patients with acute respiratory distress syndrome: A computational investigation. Crit Care Med 2020; 48:1001-1008

17. Hardman JG, Aitkenhead AR: Estimating alveolar dead space from the arterial to end-tidal $\mathrm{CO}(2)$ gradient: A modeling analysis. Anesth Analg 2003; 97:1846-1851

18. Marshall BE, Clarke WR, Costarino AT, et al: The dose-response relationship for hypoxic pulmonary vasoconstriction. Respir Physiol 1994; 96:231-247

19. The Acute Respiratory Distress Syndrome Network: Ventilation with lower tidal volumes as compared with traditional tidal volumes for acute lung injury and the acute respiratory distress syndrome. $N$ Engl J Med 2000; 342:1301-1308

20. Lang M, Som A, Mendoza DP, et al: Hypoxaemia related to COVID-19: Vascular and perfusion abnormalities on dual-energy CT. Lancet Infect Dis 2020 Apr 30. [online ahead of print]

21. Albarello F, Pianura E, Di Stefano F, et al; COVID 19 INMI Study Group: 2019-novel coronavirus severe adult respiratory distress syndrome in two cases in Italy: An uncommon radiological presentation. Int J Infect Dis 2020; 93:192-197

22. Bellani G, Laffey JG, Pham T, et al: Epidemiology, patterns of care, and mortality for patients with acute respiratory distress syndrome in intensive care units in 50 countries. JAMA 2016; 315:788-800

23. Pan C, Chen L, Lu C, et al: Lung recruitability in SARS-CoV-2 associated acute respiratory distress syndrome: A single-center, observational study. Am J Respir Crit Care Med 2020; 201:1294-1297

24. Mauri T, Spinelli E, Scotti E, et al: Potential for lung recruitment and ventilation-perfusion mismatch in patients with the acute respiratory distress syndrome from coronavirus disease 2019. Crit Care Med 2020; 48:1129-1134

25. Beloncle FM, Pavlovsky B, Desprez C, et al: Recruitability and effect of PEEP in SARS-Cov-2-associated acute respiratory distress syndrome. Ann Intensive Care 2020; 10:55

26. Roesthuis L, van den Berg M, van der Hoeven H: Advanced respiratory monitoring in COVID-19 patients: Use less PEEP! Crit Care 2020; 24:230

27. UK Intensive Care National Audit \& Research Centre Report on COVID19 in Critical Care. 2020

28. Amato MB, Meade MO, Slutsky AS, et al: Driving pressure and survival in the acute respiratory distress syndrome. N Engl J Med 2015; 372:747-755

29. Guérin C, Reignier J, Richard JC, et al; PROSEVA Study Group: Prone positioning in severe acute respiratory distress syndrome. $N$ Engl J Med 2013; 368:2159-2168

30. Carsetti A, Damia Paciarini A, Marini B, et al: Prolonged prone position ventilation for SARS-CoV-2 patients is feasible and effective. Crit Care $2020 ; 24: 225$ 